\title{
Organic Thin Film Transistors Based on Distyryl-Oligothiophenes: Role of AFM Images in Analyses of Charge Transport Properties
}

\author{
Noriyuki Yoshimoto $^{1}$, Hugues Brisset ${ }^{2,3}$, Jörg Ackermann ${ }^{2}$, Christine Videlot-Ackermann ${ }^{2 *}$ \\ ${ }^{1}$ Graduate School of Engineering, Iwate University, Morioka, Japan \\ ${ }^{2}$ Aix Marseille Université, Marseille, CNRS, CINaM UMR 7325, France \\ ${ }^{3}$ Université de Toulon MAPIEM, EA 4323, La Carde, France \\ Email: *videlot@cinam.univ-mrs.fr
}

Received October 2, 2012; revised November 1, 2012; accepted November 11, 2012

\begin{abstract}
Significant advances have been made recently in the area of organic electronics and optoelectronics based on small molecules as a result of an improved chemistry and a better technology. Together with light emitting diodes and solar cells, transistors are among the most studied components. The development of new semiconductors induced a real improvement in organic thin film transistor's performances. Additionally, the synthesis of new soluble and air-stable molecules with the ability to process the active materials at low temperatures over large areas on substrates such as plastic or paper provide unique technologies and generate new applications. However the control of the solid state structure has emerged as essential to realize the full intrinsic potential that organic semiconductors possess. Atomic force microscopy (AFM) was likely to contribute to a further advancement of knowledge. The ability of the AFM to produce three dimensional maps at the micro- and nanometer scale has greatly increased its popularity as an imaging tool. Recently, distyryl-oligothiophenes and their derivatives appear as a new class of molecular semiconductors. Detailed morphological studies of organic active layers based on such new semiconductors involved in organic thin film transistors (OTFTs) have brought a large knowledge about the impact of chemical and physico-chemical aspects on charge transport efficiency.
\end{abstract}

Keywords: Oligomer; Thin Film; Transistor; AFM

\section{Introduction}

After the initial invention of the AFM in 1986 by Binnig and Quate [1] there was a great effort focused on developing AFM instrumentation. AFM is rapidly becoming a standard microscopy technique for visualizing and measuring a material's surface structure in the physical sciences. Furthermore, AFM presents a great advantage as little or no sample preparation is required. The types of structures that are scanned with the AFM include: surfaces of bulk materials, thin films, and nanostructures that are located on a surface. There are a large number of materials that may be imaged with an AFM, including polymers, ceramics, metals, crystals, and minerals. The scan ranges for imaging in the physical sciences is from a few nanometers all the way up to tens of microns. From 1990 to 2000 applications for the AFM moved from fundamental physics to most areas of science and technology. It is estimated that in 2006 there are approxi-

*Corresponding author. mately 10,000 AFM's in use around the world. The AFM is now utilized in a much wider area than others techniques as scanning probe microscopy (STM), from basic biology through to semiconductor production lines, because of the lower restrictions on the sample structures.

Organic electronics saw the day with the discovery in the late 1970s of the first "conductive polymer" in its doped form which is otherwise a semiconductor. Nobel Laureates in Chemistry in 2000, A. J. Heeger, A. G. MacDiarmid and H. Shirakawa were awarded for their revolutionary discovery that plastic, through some modifications, can be made electrically conductive. Electronics based on organic semiconductors has evolved from constant and large efforts in the field of materials, processing and circuit design. Together with light emitting diodes and solar cells, transistors are among the most studied components. While development of new semiconductors based on improved chemistry and on better technology induced a real improvement in device's performances, a detailed study of the involved active 
layers is necessary not only on an electrical point of view but also from a morphological aspect. Analogous to conventional inorganic semiconductors, the performance of organic semiconductors is directly related to their molecular structure and packing, crystallinity, growth mode, and purity. In order to achieve the best possible performance, it is critical to understand how organic semiconductors grow. As a great importance is done to the relationship between electrical performances and morphology, AFM is likely to contribute to a further advancement of knowledge. The AFM since its introduction has gained wide usage in surface topography measurements at the very small scale. The ability of the AFM to produce three dimensional maps at the micro- and nanometer scale has greatly increased its popularity as an imaging tool of thin films. Unlike polymers, molecular materials, called small organic molecules, have advantages such as well-defined structures, easier purification and more easily controllable properties. Such organic materials can be deposited on substrates by different processes to form thin films as active layers in organic thin film transistors (OTFTs). Despite different materials (metal, insulator or semiconductors) are involved in OTFTs, the morphology of the active layer based on such organic semiconductors on measured performances is one of the key parameters. After a description of AFM as an imaging technique to characterize such organic active layers involved in OTFTs, we will present the most OTFT configurations or architectures commonly used in the literature together with the electrical operating mode of transistor devices. The series of distyryl-oligothiophenes and their derivatives were recently presented as a novel class of OTFT semiconductors. In the present paper, a direct impact of their thin film morphology on charge transport efficiency is highlighted by AFM where the molecular structure, the deposition process, the substrate nature and the thin film thickness are some of direct parameters.

\section{Description of AFM as an Imaging Technique}

The AFM consists of a cantilever with a sharp tip (probe) at its end that is used to scan the surface. The cantilever is typically silicon or silicon nitride with a tip radius of curvature on the order of nanometers. When the tip is brought into proximity of a sample surface, forces between the tip and the sample lead to a deflection of the cantilever according to Hooke's law. Depending on the situation, forces that are measured in AFM include mechanical contact force as van der Waals forces, capillary forces, chemical bonding, electrostatic forces, magnetic forces. Typically, the deflection is measured using a laser spot reflected from the top surface of the cantilever into an array of photodiodes. If the tip was scanned at a con- stant height, a risk would exist that the tip collides with the surface, causing damage. Hence, in most cases a feedback mechanism is employed to adjust the tip-tosample distance to maintain a constant force between the tip and the sample. Traditionally, the sample is mounted on a piezoelectric tube, which can move the sample in the $z$ direction for maintaining a constant force, and the $x$ and $y$ directions for scanning the sample. Alternatively a "tripod" configuration of three piezo crystals may be employed, with each responsible for scanning in the $x, y$ and $z$ directions. This eliminates some of the distortion effects seen with a tube scanner. In newer designs, the tip is mounted on a vertical piezo scanner while the sample is being scanned in $x$ and $y$ using another piezo block. The resulting map of the area $z=f(x, y)$ represents the topography of the sample.

The AFM can be operated in a number of modes, depending on the application. In general, possible imaging modes are divided into static (also called contact) modes and a variety of dynamic (non-contact or "tapping") modes where the cantilever is vibrated.

- Contact mode: Contact mode is the most basic operation mode to observe topographic images of samples. Because the tip directly contacts with the sample surface in contact mode, damage by friction force between the tip and sample becomes problems. The static deflection of cantilever is measured by detecting the position of reflected laser beams, and used as a feedback signal for controlling the force given to the tip. The force between the tip and the surface is kept constant during scanning by maintaining a constant deflection.

- Tapping mode: Tapping mode was developed in 1990s, and overcomes the problems peculiar to contact mode to obtain high-resolution topographic images of materials without any damaging. In tapping mode, the cantilever is driven to oscillate at its resonance $(50 \mathrm{k}-500 \mathrm{kHz})$. When the tip approaches to sample surface, the amplitude of oscillation decreases due to repulsion interaction between the tip and the sample. Keeping the amplitude to be constant by a feedback loop during scan across the surface, topographic images of samples are obtained from the amplitude signal. Tapping mode overcomes problems associated with friction that a plague conventional contact method by alternately placing the tip in contact with the surface to provide high resolution and then lifting the tip off the surface to avoid dragging of samples by friction between the tip and the surface. Most of the extremely high resolution images measured with an AFM in ambient air are made with tapping mode. The image represents the amplitude variation of the square root of the amplitude (rms). The resolution is somewhat worse than in the contact 
mode but molecular resolution can still sometimes be obtained. The characterization by AFM in tapping mode is used to determine several parameters as the growth mode, the surface layer roughness, the grain size of polycrystalline thin film deposited on substrates, the presence and the nature of defects as grain boundaries, cracks, and dislocations.

\section{Organic Thin Film Transistors (OTFTs): Description and Characterization}

OTFTs are desired for the manufacture of low-cost electronic devices involving in modern electronics such as smart cards, RFID tags, flexible electronic paper, and backplane circuitry for active matrix displays [2-4]. While must of the attention of the organic transistors community has been focused on the search for high-mobility, ambient stable, and solution-processable small molecule and polymeric semiconductor materials, it is now admitted that substantial improvements in OTFT performance is also obtained by focusing on the morphology of the organic active layer involved in charge transport. A field effect transistor consists of a semiconducting layer based on an organic semiconductor (OSC), a dielectric layer and three electrodes, namely gate $(\mathrm{G})$, source (S) and drain (D). Silicon dioxide $\left(\mathrm{SiO}_{2}\right)$, the oxide of silicon, together with silicon are the most widely used dielectric and gate materials, respectively. The most often process to grow silicon dioxide on the surface of silicon wafers is the thermal oxidation where the silicon is exposed to oxidizing agents such as water and oxygen at elevated temperatures. This process has good control over the thickness and properties of the $\mathrm{SiO}_{2}$ layer. Such resulting bilayers are commonly named $\mathrm{Si} / \mathrm{SiO}_{2}$ substrates. There exist four device configurations where the bottom gate (BG) configurations, with either top or bottom contact (TC or $\mathrm{BC}$ ) as shown in Figure 1, are espe- cially suitable for transistors with $\mathrm{SiO}_{2}$ dielectric layer. In such cases the OSC-based layer is deposited on top of the dielectric layer, after its growth and modification of its surface. Thus, minimal risk exists that the technological processes may negatively influence the structure or morphology of the semiconductor layer or lead to a partial decomposition of the semiconductor. Several techniques are used to deposit the OSC layer as vapour phase under vacuum or liquid-based process as spin coating and drop-casting. In the first case, the semiconductor is vaporized and then condenses into a film onto a substrate. Some parameters as the rate of evaporation, the substrate temperature $\left(\mathrm{T}_{\text {sub }}\right)$, the thickness can be controlled. For solution deposition, the organic semiconductor is completely dissolved in an organic solvent. The solution is then coated onto the substrate by a liquid-based process. As the solvent vaporizes, the solution becomes super-saturated and forms organic semiconductor layers.

A field-effect transistor operates as a voltage-controllled current source. By applying the gate voltage $\left(\mathrm{V}_{\mathrm{G}}\right)$ across the gate dielectric, a sheet of mobile charge carriers is induced in the semiconductor that allows a current (the drain current $I_{D}$ ) to flow through the semiconductor when another voltage (the drain-source voltage $\mathrm{V}_{\mathrm{DS}}$ ) is applied between drain and source. The charge transport, operating by a hopping process in OTFT devices, occurs in the OSC layer near the dielectric which is called OSC/dielectric interface on Figure 1. In electrical operating mode of transistor devices, there are three crucial parameters. These are the charge carrier mobility ( $\mu_{\mathrm{e}}$ or $\mu_{\mathrm{h}}$ for electron or hole mobilities), the threshold voltage $\left(\mathrm{V}_{\mathrm{T}}\right)$ and the Ion/Ioff ratio.

They can be determined from so called output and transfer characteristics of the transistor (Figure 2). Transistor activity is observed by application of negative or positive drain and gate voltages to operate in the accumulation
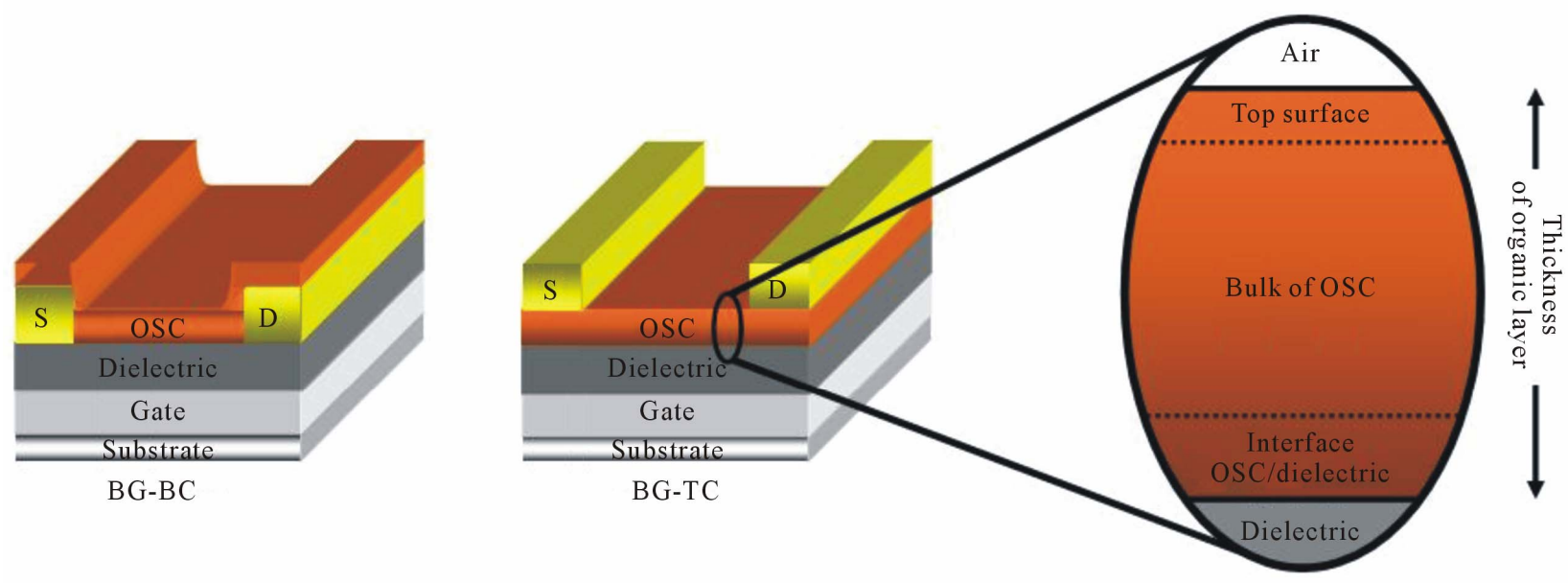

Figure 1. Bottom gate (BG) configurations of organic thin film transistors with bottom (BC) or top (TC) source-drain contacts. Zoom-in of the organic active layer. 


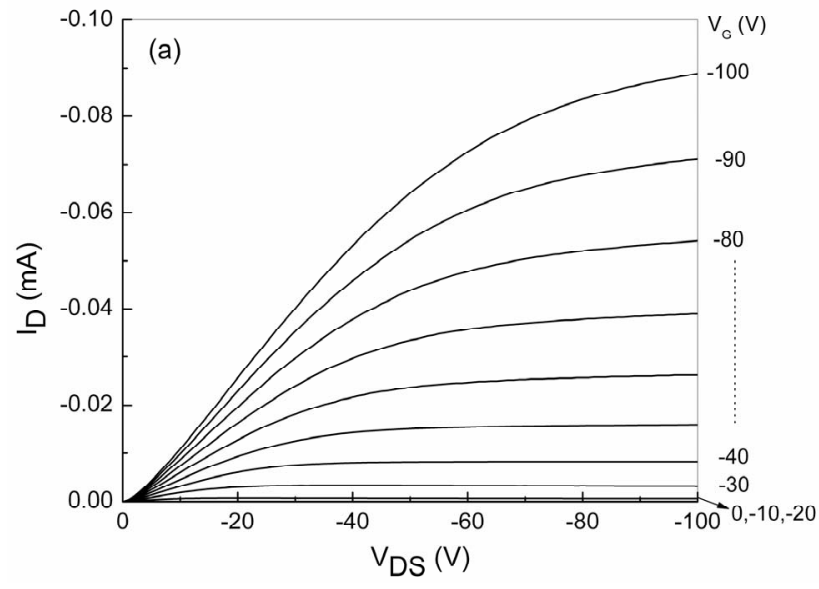

(a)

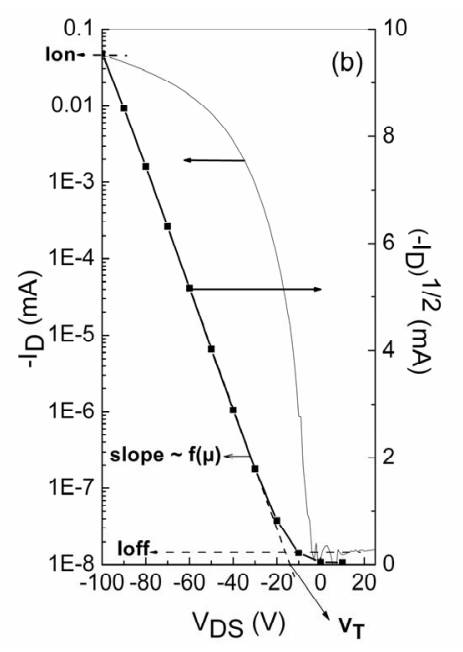

(b)

Figure 2. Typical output (a) and transfer (b) characteristics of $p$-channel OTFTs.

mode meaning that the organic molecules involved in the active layer behave as $p$ - or $n$-type semiconductors, respectively.

Many of $n$-channel materials only oprate in vacuum or under an inert atmosphere due to electron trapping by ambient oxygen and moisture. Even if several new $n$-channel semiconductors have exhibited high performances together with high stability in ambient conditions [5], it is still challenging to fabricate high-performing ambient-stable $n$-channel OTFTs. Like $p$-channel OTFTs, electron transport in $n$-channel thin films is greatly governed by their morphology and molecular orientation where key parameters are thin-film growth conditions. Most issues such as electronic performances and air stability have been greatly influenced by quality thin films where highly oriented polycrystalline thin films exhibit higher charge-carrier mobility than amorphous films or randomly oriented crystalline films. It is well-known that the ordering of organic molecules in the anchoring region near the dielectric layer (interface OSC/dielectric on Figure 1) are strongly affected by the substrate surface structure and the balance of molecule-molecule and molecule-substrate interactions. Therefore, the use of visualization techniques becomes important to observe the morphology of the active layer together with its different parameters. Most of organic molecular films are electrically insulating and can therefore be investigated by AFM and related techniques using some force interactions between the scanned probe and the sample surface such as van der Waals, frictional, electrostatic, capillary and imaging forces. Because of the very different physical nature of the two media, OSC and dielectric, the deposition techniques as vapour phase under vacuum, should result in highly disordered films, leading to very poor performance. However a good organization can be obtained by heating the substrate at different $\mathrm{T}_{\text {sub }}$ and depositing the organic semiconductor at a lower rate as commonly observed for organic thin films based on small molecules as pentacene, oligothiophene derivatives and many others. Additionally, different parameters can also highly influence the resulting performances of active layers as the molecular structure, the deposition process, the substrate nature and the thin film thickness.

\section{Distyryl-Oligothiophenes and Derivatives: A Novel Class of OTFT Semiconductors}

Long range interconnected crystalline grains with small grain boundary are favorable to charge transport as observed in pentacene-based OTFTs [6,7]. Oligothiophenes, distyryl-oligothiophenes and their derivatives do not derogate from the rule. A new series of structurally simple and readily available oligothiophenes end-capped with styryl units, named distyryl-oligothiophenes DS $n$ Ts $(n=$ 2 - 4), represents a novel class of OTFT semiconductors that combine good electrical performances and exceptional stabilities [8]. Transistor responses were obtained only for negative bias, which is indicative that DSnT compounds behave as $p$-type semiconductors in air. The field effect mobilities calculated in the saturation regime are shown to increase with the substrate deposition temperature $\left(T_{\text {sub }}\right)$ as due to the formation of better ordered thin films at elevated $\mathrm{T}_{\text {sub }}$. The highest value of the hole mobility $\left(0.1 \mathrm{~cm}^{2} / \mathrm{Vs}\right)$ was obtained for the longest oligomer DS4T (see molecular structure on Figure 3) at $110^{\circ} \mathrm{C}$ (Table 1). Additionally, DS4T-based OTFTs were also found to possess an exceptional long-lifetime (more than one year) and an electrical stability toward continuous operation.

The morphology of vapor deposited thin films of DS4T grown on $\mathrm{SiO}_{2}$ was investigated using AFM where 
Table 1. OTFT data of DSnTs deposited at different substrate temperatures $\left(\mathrm{T}_{\text {sub }}\right)$ on $\mathrm{SiO}_{2}$ layers in BG-TC configuration [8].

\begin{tabular}{ccccc}
\hline Material & $\mathrm{T}_{\text {sub }}\left({ }^{\circ} \mathrm{C}\right)$ & $\mu\left(\mathrm{cm}^{2} / \mathrm{Vs}\right)$ & Ion/Ioff & $\mathrm{V}_{\mathrm{T}}(\mathrm{V})$ \\
\hline DS2T & 30 & $0.002-0.006$ & $1.6-1.9 \times 10^{5}$ & $4-8$ \\
& 80 & $0.01-0.02$ & $2-2.3 \times 10^{5}$ & $0-19$ \\
DS3T & 30 & $0.001-0.008$ & $2.2-2.8 \times 10^{4}$ & $0.1-3$ \\
& 80 & $0.01-0.02$ & $2.2-3.3 \times 10^{4}$ & $4-13$ \\
DS4T & 30 & $0.02-0.04$ & $1.2-1.8 \times 10^{3}$ & $3-13$ \\
& 80 & $0.03-0.06$ & $1.9-2.5 \times 10^{3}$ & $12-20$ \\
& 110 & $0.08-0.1$ & $0.8-1.2 \times 10^{3}$ & $5-20$ \\
\hline
\end{tabular}

the formation of islands whose size increases with the substrate deposition temperature was observed. At $\mathrm{T}_{\text {sub }}=$ $110^{\circ} \mathrm{C}$, a terrace-and-step morphology with small grain boundary is clearly observed, an average value of $2.9 \mathrm{~nm}$ being determined for the steps height and associated to "up-right molecules" (Figure 3). The overlap of $\pi$ orbitals between grains containing up-right oriented molecules is by consequence favorable to an efficient carrier path charge at the grain boundary to ensure an efficient charge transport in OTFTs.

Recently, from thin films based on a "kite" shaped styryl end-capped benzo[2,1-b:3,4- $b$ '] dithiophene (KDS2T) emerges also an obvious correlation between the grain size and OTFT performances according to the substrate temperature $\left(\mathrm{T}_{\text {sub }}=30^{\circ} \mathrm{C}\right.$ or $\left.80^{\circ} \mathrm{C}\right)$ [9]. The molecular structure of KDS2T is shown in Figure 4. While a

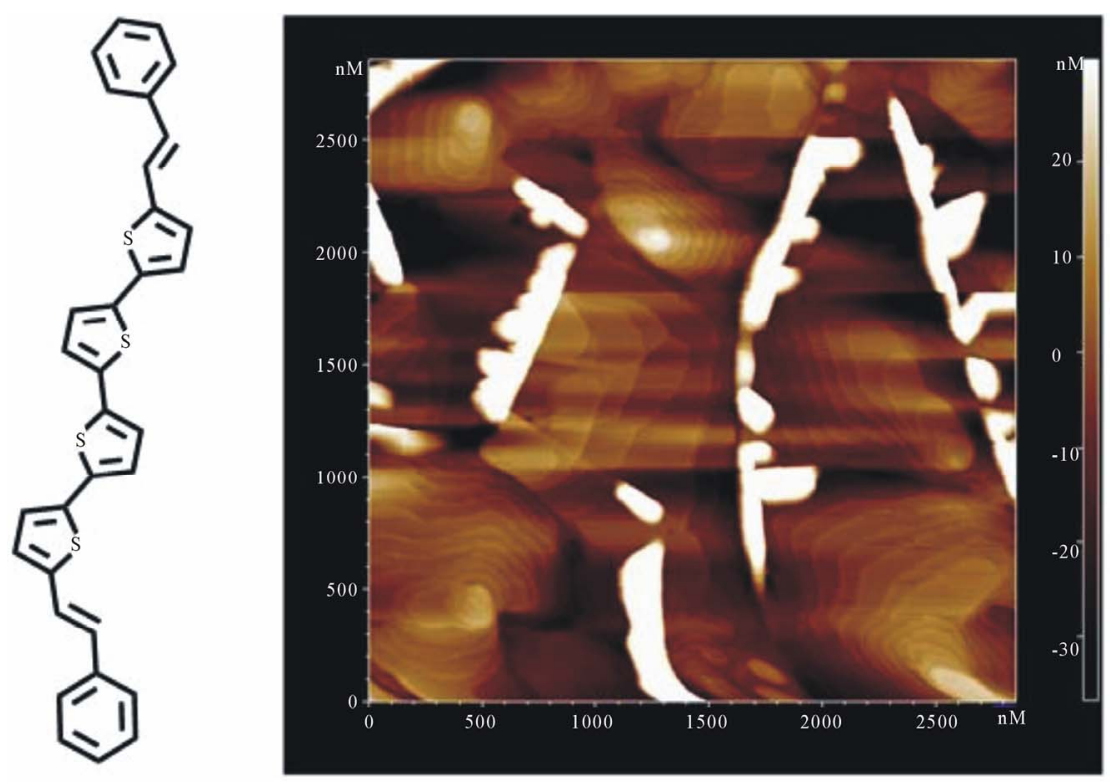

Figure 3. AFM image of a DS4T (molecular structure on the left side) film deposited at $\mathrm{T}_{\text {sub }}=110^{\circ} \mathrm{C}$ on a $\mathrm{Si} / \mathrm{SiO} \mathrm{O}_{2}$ substrate with a nominal thickness of $50 \mathrm{~nm}[8]$.
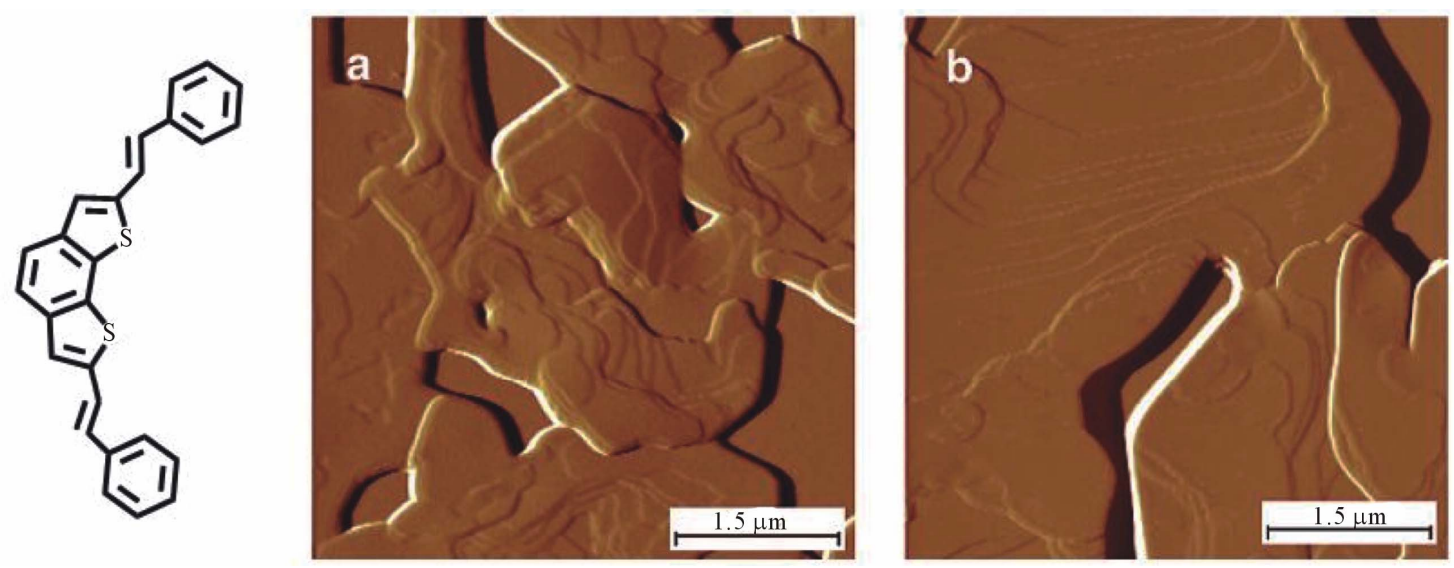

Figure 4. AFM images of KDS2T (molecular structure on the left side) films deposited on $\mathrm{Si} / \mathrm{SiO}_{2}$ substrates at $\mathrm{T}_{\text {sub }}=30^{\circ} \mathrm{C}(\mathrm{a})$ and $80^{\circ} \mathrm{C}(\mathrm{b})$ with a nominal thickness of $50 \mathrm{~nm}$ [9]. 
terrace-like step structure was observed for both $\mathrm{T}_{\text {sub }}$ values together with a comparable grain boundary length, small crystal grains of $1.5-3 \mu \mathrm{m}$ in size are observed in the AFM images of thin films obtained at $30^{\circ} \mathrm{C}$ (Figure 4(a)) and the grain size increases to $\sim 3-7 \mu \mathrm{m}$ at $\mathrm{T}_{\text {sub }}=$ $80^{\circ} \mathrm{C}$ as shown in Figure 4(b). In the present study, a direct correlation is established between on increased field effect mobility calculated in the saturation regime with the substrate deposition temperature where ordered thin films with larger grains are formed at higher temperatures.

Additionally to the control of the substrate temperature, the fine control of the thin film thickness can provide further information as previously observed for pentacene-based thin films [6,7]. As an example, Figure 5 shows AFM images of thin films based on an oligomer (DFH-4T) deposited by vacuum evaporation on $\mathrm{Si} / \mathrm{SiO}_{2}$ substrates heated to $50^{\circ} \mathrm{C}$ or $80^{\circ} \mathrm{C}$ to a nominal thickness of $3,5,15$ or $30 \mathrm{~nm}$ [10]. While separate islands with dendritic shapes are observed at $\mathrm{T}_{\text {sub }}=50^{\circ} \mathrm{C}$ (Figures 5(a) and (b)) following an island growth mode, AFM images at $\mathrm{T}_{\text {sub }}=80^{\circ} \mathrm{C}$ show larger and interconnected grains with a more homogenous distribution in size and height. Figures 5(c) and (d) demonstrate an almost complete covering of the substrate surface by the first DFH-4T monolayer (ML) as well as the successive ML formation. The growth mode switch to a layer-to-layer growth mode for DFH-4T films grown on $\mathrm{SiO}_{2}$ substrates heated to $80^{\circ} \mathrm{C}$, revealing the strong impact of the substrate temperature on the film morphology. At low substrate temperature (typically $30^{\circ} \mathrm{C}$ ) during the deposition of molecules, an electron mobility value of $1 \times 10^{-5} \mathrm{~cm}^{2} / \mathrm{V}$.s has been obtained. As already observed for OTFTs based on $\mathrm{DS} n \mathrm{Ts}$, the field effect mobilities calculated in the saturation regime are shown to increase with the substrate deposition temperature as due to the formation of better ordered thin films at elevated $\mathrm{T}_{\text {sub }}$. An electron-mobility
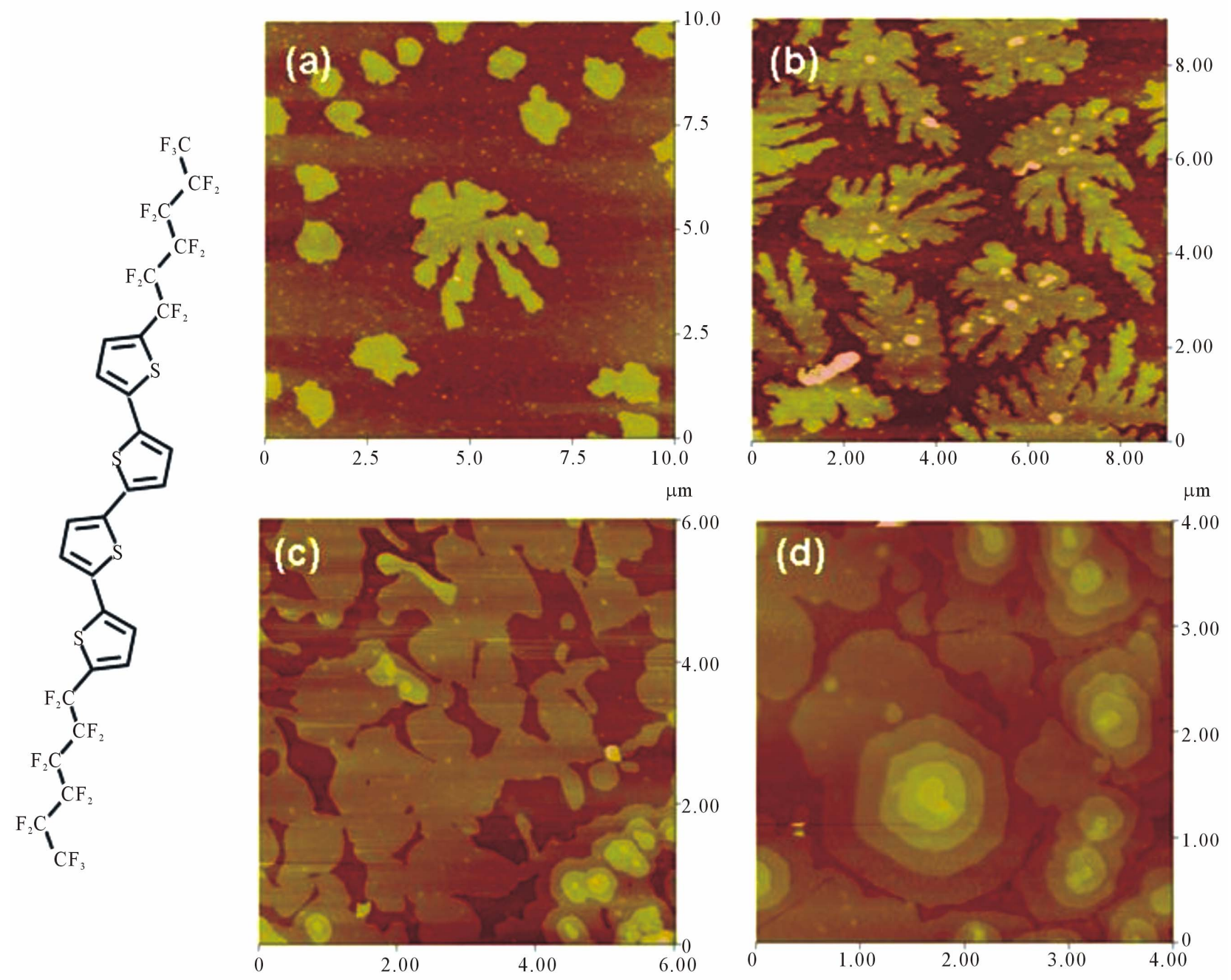

$\mu \mathrm{m}$

$\mu \mathrm{m}$

Figure 5. AFM images of DFH-4T (molecular structure of DFH-4T on the left side) films deposited on $\mathrm{Si} / \mathrm{SiO}_{2}$ substrates at $T_{\text {sub }}=50^{\circ} \mathrm{C}(\mathrm{a}, \mathrm{b})$ and $\mathrm{T}_{\text {sub }}=80^{\circ} \mathrm{C}(\mathrm{c}, \mathrm{d})$ with a nominal thickness of $3 \mathrm{~nm}(\mathrm{a}), 5 \mathrm{~nm}(\mathrm{~b}), 15 \mathrm{~nm}$ (c) and $30 \mathrm{~nm}$ (d) [10]. 
up to $6 \times 10^{-4} \mathrm{~cm}^{2} / \mathrm{V}$.s for DFH-4T films deposited at $80^{\circ} \mathrm{C}$ on a dielectric layer based on polymethylmethacrylate (PMMA) was measured in air. The difference of one order in the mobility of DFH-4T between $\mathrm{T}_{\text {sub }}=30^{\circ} \mathrm{C}$ and $\mathrm{T}_{\text {sub }}=80^{\circ} \mathrm{C}$ can be directly correlated to the grain size determined in the morphology study, where DFH-4T showed larger grains on heated substrates due to its layer-to-layer growth mode. Furthermore, while most of the results reported on transistors incorporating DFH-4T thin films prepared by vacuum deposition on silicon dioxide $\left(\mathrm{SiO}_{2}\right)$, hexamethyldisilazane (HMDS), poly(styrene) (PS) and poly (vinyl alcohol) (PVA) dielectric layers [11] where measured in vacuum, an independent work has demonstrated relatively air-stable $n$-channel DFH-4T based transistors by using PMMA as the organic insulating layer [10]. Despite lower electron carrier mobility, such the strategy to modify the insulator surface properties by PMMA to eliminate electron trapping sites could give rise to operational transistors in ambient conditions.

AFM study on organic thin films can also highlighted the influence of molecular structure on growth mode. While a layer-by-layer growth together with an island one, characteristic of the Stranski-Krastanov mechanism, was observed for DS3T based thin films on heated substrates, the formation of the islands in diPhAc-3T thin films is achieved by the Volmer-Weber mechanism, characteristic of a 3-dimensional (3D) growth (Figure 6) $[12,13]$. The growth of thin films is here essentially controlled by two types of interaction, the interaction between molecules and substrate and the intermolecular interaction which can be modulated by heating the substrate during the solid state formation of organic thin films. These results underline the importance of molecular structure on growth mechanism and resulting thin film morphology for electronic applications such as charge transport in OTFTs. Together with high performing OTFTs elaborated at low $\mathrm{T}_{\text {sub }}$ i.e. $25^{\circ} \mathrm{C}-50^{\circ} \mathrm{C}$, di-
PhAc-3T based thin films benefit from a compact solid state to block the introduction of air containing oxidizing contaminants conferring high air stability to OTFTs over the time [13].

Furthermore, the efficient cohesion of diPhAc-3T vacuum evaporated thin films induced by a $3 \mathrm{D}$ growth offers an exceptional high physical resistance to a laser pulse. Active layers based on diPhAc-3T for BG-TC transistors have been printed using the laser induced forward transfer (LIFT) technique [14]. diPhAc-3T was vacuum evaporated on a quartz substrate prior a transfer by laser on an acceptor substrate (typically a $\mathrm{Si} / \mathrm{SiO}_{2}$ substrate) to form an organic active layer for charge transport. Resulting printed diPhAc-3T pixels on receiver substrates have well defined morphological properties as shown by optical microscopy and AFM (Figure 7). Electrical characterizations demonstrated that transistors are fully operative with hole mobilities up to $0.04 \mathrm{~cm}^{2} / \mathrm{V}$.s, threshold voltage $\mathrm{V}_{\mathrm{T}}$ near $0 \mathrm{~V}$ and Ion/Ioff ratio up to $2.8 \times$ $10^{5}$. The high intermolecular interaction involved in such growth mechanism makes thin films weakly sensitive to the mechanical damages induced by the laser. These results underline the importance of molecular structure on growth mechanism and resulting thin-film cohesion for the realization of laser printed OTFTs.

In the oligothiophene series, the $n$-type semiconducting counterparts have been obtained by end-substitution of the oligomer backbone with electron withdrawing groups (EWGs) as perfluoroarenes. The synthesis and the solid-state properties of two new perfluoroarene-terminated oligothiophene derivatives DFS $n$ Ts $(n=2,4)$ were reported recently $[15,16]$. The molecular structure of DFS2T and DFS4T are shown on Figure 8. Actually, these compounds are the perfluorinated analogues of $\alpha, \omega$-distyryl-oligothiophenes, DSnTs ( $\mathrm{n}=2-4)$, which exhibited $p$-type semiconducting properties and high stability in OTFTs [8]. With DFSnTs, both $n$-type carrier
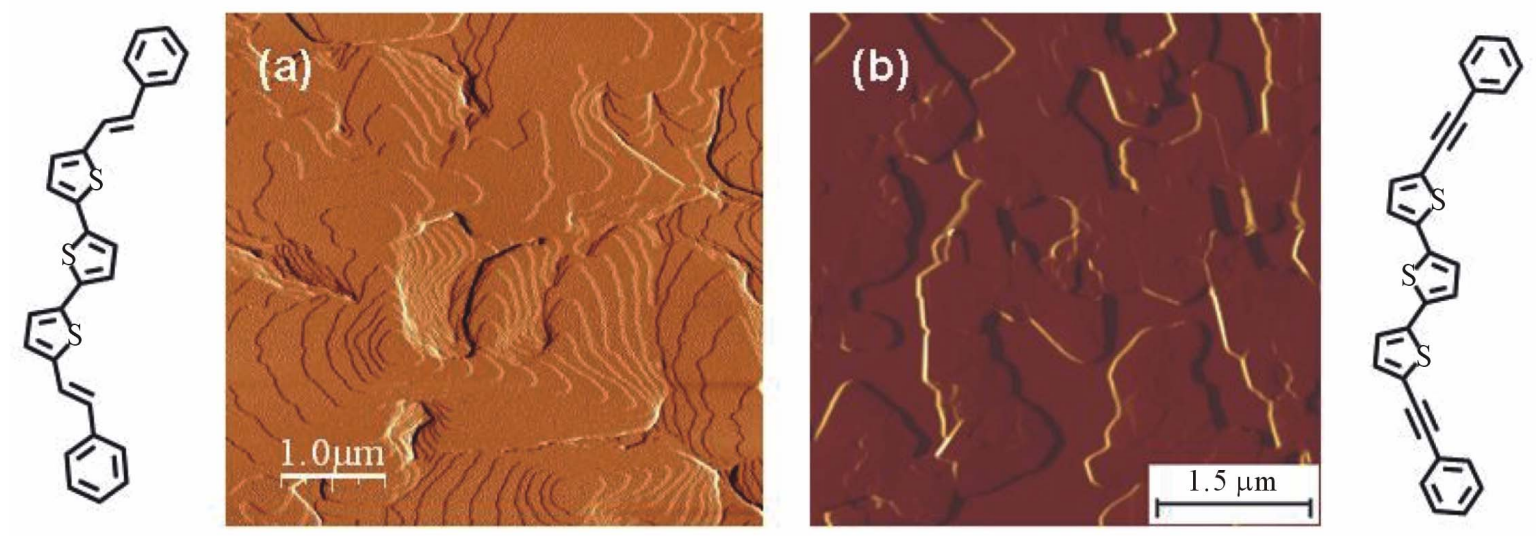

Figure 6. AFM images of DS3T and diPhAc-3T-based thin films with a nominal thickness of $50 \mathrm{~nm}$ deposited on $\mathrm{Si} / \mathrm{SiO}_{2}$ heated substrates $\left(T_{\text {sub }}=80^{\circ} \mathrm{C}\right.$ for DS3T in (a) and $T_{\text {sub }}=50^{\circ} \mathrm{C}$ for diPhAc-3T in (b)) $[12,13]$. Molecular structures of DS3T and diPhAc-3T on both side of corresponding AFM images. 

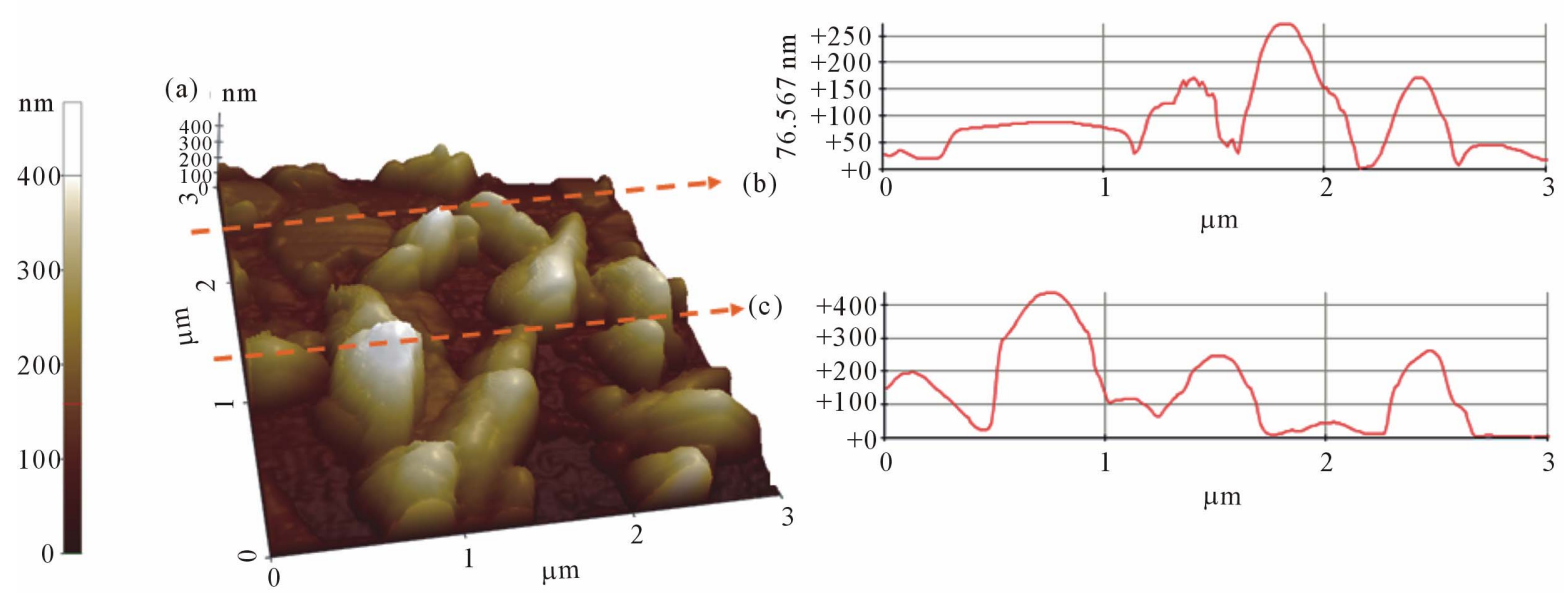

$\mu \mathrm{m}$

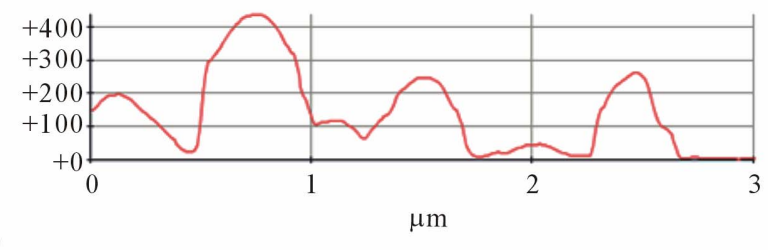

Figure 7. AFM image (a) and cross-sections (b), (c) of the surface of a diPhAc-3T-based pixel printed by LIFT at $0.26 \mathrm{~J} / \mathrm{cm}^{2}$ [14].
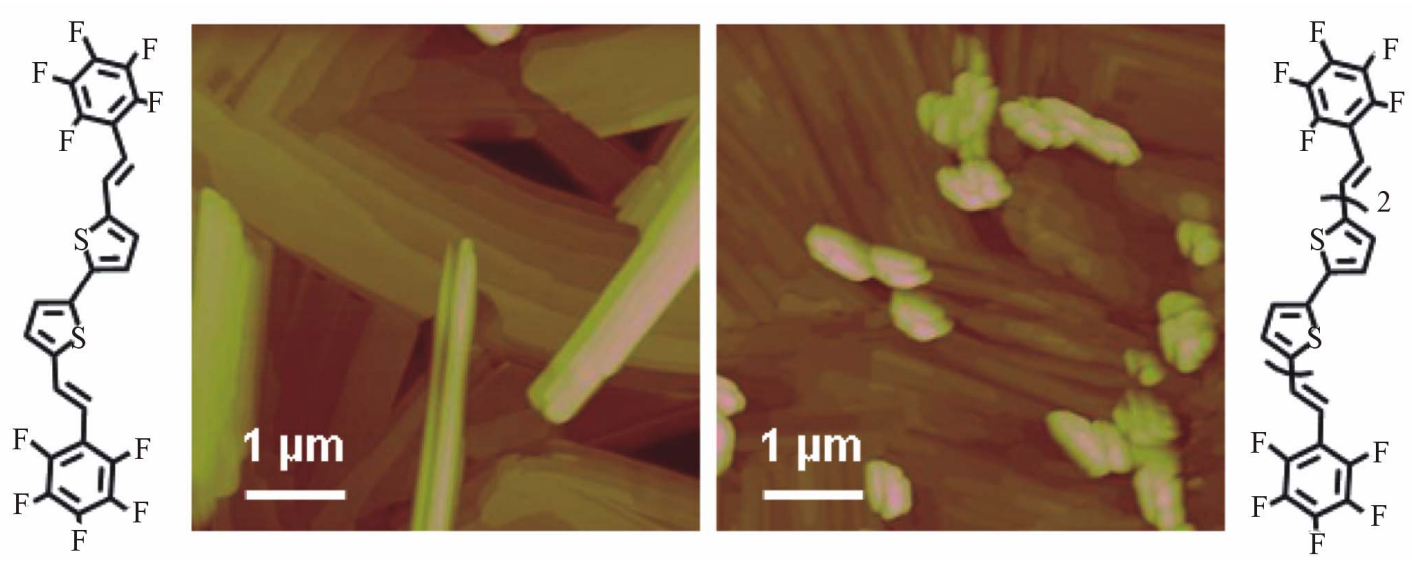

Figure 8. AFM pictures of DFS2T and DFS4T thin films deposited with a nominal thickness of $50 \mathrm{~nm}$ on $\mathrm{Si} / \mathrm{SiO} \mathrm{O}_{2} \mathrm{substrates}$ heated to $T_{\text {sub }}=80^{\circ} \mathrm{C}$ [16]. Molecular structures of DFS2T and DFS4T on both side of corresponding AFM images.

dominance and remarkable attributes characterizing the DS $n$ Ts have been expected to be maintained. Surprisingly, both compounds, DFS2T and DFS4T, do not show $n$-type transport but were $p$-type materials for OTFT measurements realized in air and in vacuum. By using a controlled low evaporation rate together with a fine control of the substrate temperature [16], highly interconnected $\mu \mathrm{m}$-long rodlike crystallites were obtained at $\mathrm{T}_{\text {sub }}=80^{\circ} \mathrm{C}$ (Figure 8). Such observation by AFM has removed the doubt as a lack of electron transport in organic-based thin films is due to a poor morphology as suggested by Tang and Bao [17]. Analyses of these materials reveal a direct impact of the molecular structure where the presence of the two double bonds may affect the final transport properties by confining the electrons in some segments of the molecular backbone bonds thus thwarting the $\pi$-conjugation [15].

As efficient EWGs, some substitutions by either alkyl groups $\left(-\mathrm{CF}_{3}\right)$ on aryl naphthalenetetracarboxylic diimide (NTCDI) enable to lower LUMO level and help to im- prove electron charge injection. Resulting mobilities and air stability on $n$-channel OTFTs have been considerably increased [18]. In order to expand the series of fluorinated distyryl-bithiophenes, two new perfluoroalkyl endsubstituted analogues of distyryl-bithiophene were synthesized by introducing $-\mathrm{CF}_{3}$ groups on arene end-units depending on their number and position [19]. The molecular structure of both oligomers, $\mathrm{CF}_{3}$-DS2T and $\mathrm{diCF}_{3}$-DS2T, is shown on Figure 9. A comparative study with the perfluoroarene-containing distyryl-bithiophene analogue (DFS2T) underlines the influence of perfluorination by either alkyl groups $\left(-\mathrm{CF}_{3}\right)$ or by fluorine atoms $(-\mathrm{F})$ on arene end-units of DS2T $\pi$-conjugated core. While DFS2T implemented as active layer into OTFTs behaves as a $p$-type organic semiconductor, $\mathrm{CF}_{3}-\mathrm{DS} 2 \mathrm{~T}$ leads to $n$-channel OTFTs measured in vacuum where any obvious electron density confinement in the molecular backbone occurred [19]. With a fine microstructure observed by AFM and XRD of $\mathrm{CF}_{3}$-DS2T-based thin films deposited on HMDS-treated $\mathrm{Si} / \mathrm{SiO}_{2}$ substrates 
heated to $80^{\circ} \mathrm{C}$ (Figure 9), electron injection can occur from gold electrodes to LUMO level generating an electron transport. A mobility up to $9.8 \times 10^{-4} \mathrm{~cm}^{2} / \mathrm{V}$.s together with an Ion/Ioff ratio of $\sim 10^{7}$ were measured under high vacuum. The absence of a fine solid-state ordering in diCF$_{3}-\mathrm{DS} 2 \mathrm{~T}$ thin films at either $30^{\circ} \mathrm{C}$ or $80^{\circ} \mathrm{C}$ revealed by $\mathrm{XRD}$ leads to a total absence of charge transport in such films. Analyses of these materials reveal a direct relationship between molecular structure, solid state microstructure and electrical properties. The results of this study indicate that the molecular geometry and intermolecular interactions in the crystalline state govern the electrical properties of OTFTs by directly influencing key factors as interface states and morphology of organic thin films. The main result coming out of this comparative study is the influence of the high electronic effect of $-\mathrm{CF}_{3}$ groups together with a fine molecular arrangement in $\mathrm{CF}_{3}$-DS2T-based thin films to control the charge carrier type, i.e. electron, transported in such organic active layers.

In others cases, unfavorable macroscopic morphology observed by AFM can explain the lack of charge transport in OTFTs. Solid-state properties of two new distyryl-bithiophene derivatives with cyano groups at different positions on the ethylene linkage show drastic different behavior from each other [20]. While the surface morphology of one of them as $50 \mathrm{~nm}$ thick vacuum deposited layer on $\mathrm{Si} / \mathrm{SiO}_{2}$ substrates heated at $80^{\circ} \mathrm{C}$ shows numerous needle like grains going out of the plane spaced by in-plane grains, a quasi-amorphous morphology is observed for the second one in the same conditions. All thin films of the first one are field-effect transistor active in air revealing a hole charge transport. On the contrary, thin films based on the second one show no response under either positive or negative gate voltage meaning that this compound shows neither an $n$ - nor a $p$-channel activity in air but behaves as an insulator. The comparative analysis of the electrical responses obtained under stringently identical conditions reveals the great importance of the position of cyano groups on ethylene linkage [20].

From the soluble properties of some oligomers, different techniques using OSC-based solutions can be used in addition to vapour phase under vacuum. Based on a small molecule, the $\alpha, \omega$-hexyl-distyryl-bithiophene (DHDS2T), a series of OTFT devices were realized by vapor phase, spin-coating, drop casting and inkjet printing for a comparative analysis of their electrical response/behavior obtained under identical measurement conditions [21]. For vacuum-evaporated DH-DS2T thin films, AFM pictures show a polycrystalline morphology where monolayer terraces are clearly observed with an average value of $3.2 \mathrm{~nm}$ as determined for the steps height and corresponding to the molecular length ( 3.4 - $3.5 \mathrm{~nm})$ (Figure 10(a)). Along with these grains, a large number of needles like grains pointing out of the substrates are observed. Spin-coated thin films exhibit a thin layer homogeneously dispersed on the surface together with higher grains (Figure 10(b)). Upon changing to dropcasted thin films, large domains can be observed with terrace steps of $3.2 \mathrm{~nm}$ (Figure 10(c)). Different parameters linked directly to the processes (solvent, concentration, deposition method, surface, post-treatment...) are identified as key factors controlling film quality/ crystallinity and device performances. While all OSClayers give rise to active $p$-channel OTFTs corresponding to a hole transport, such systematic study reveals the factors that limit efficient charge transport at the macroscopic scale of the channel length in OTFT devices.

\section{Conclusion}

While initial progress was mostly attributable to synthetic efforts in the form of the creation of new molecular
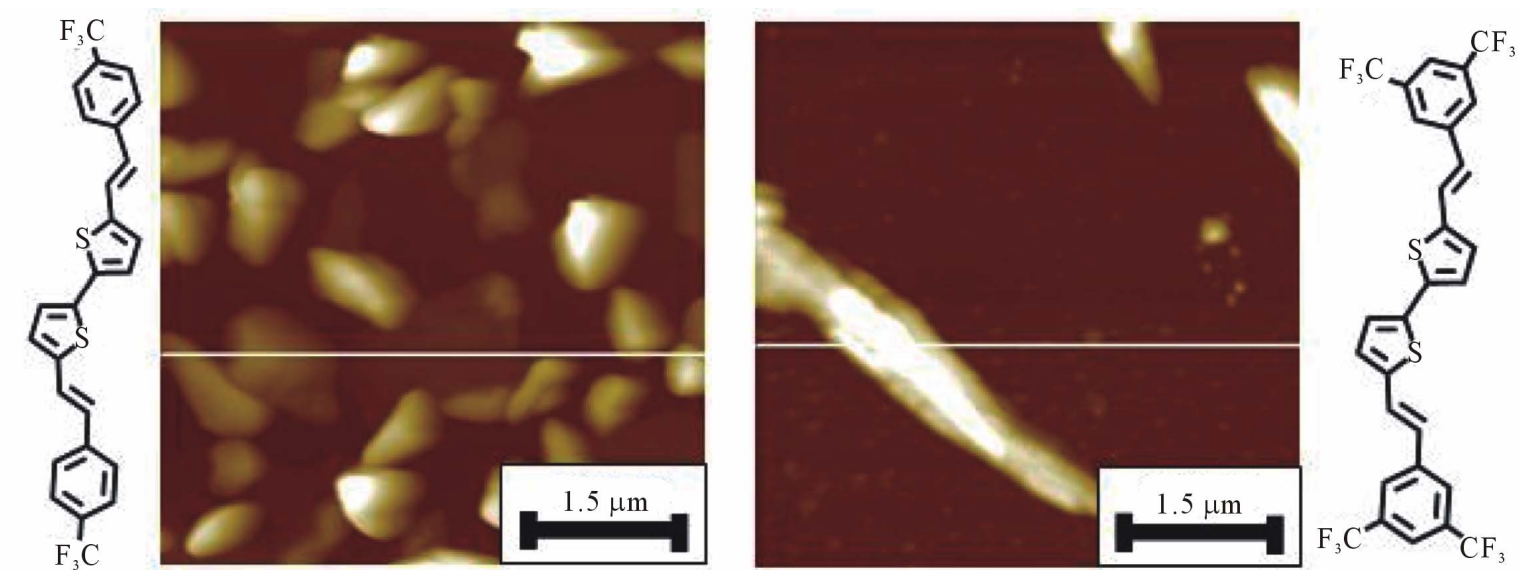

Figure 9. AFM images of $\mathrm{CF}_{3}-\mathrm{DS} 2 \mathrm{~T}$ and diCF$_{3}-\mathrm{DS} 2 \mathrm{~T}$-based thin films with a nominal thickness of $50 \mathrm{~nm}$ deposited on HMDS-treated $\mathrm{Si} / \mathrm{SiO}_{2}$ substrates heated to $\mathrm{T}_{\text {sub }}=80^{\circ} \mathrm{C}$. Molecular structures of $\mathrm{CF}_{3}-\mathrm{DS} 2 \mathrm{~T}$ and diCF ${ }_{3}-\mathrm{DS} 2 \mathrm{~T}$ on both side of corresponding AFM images [19]. 

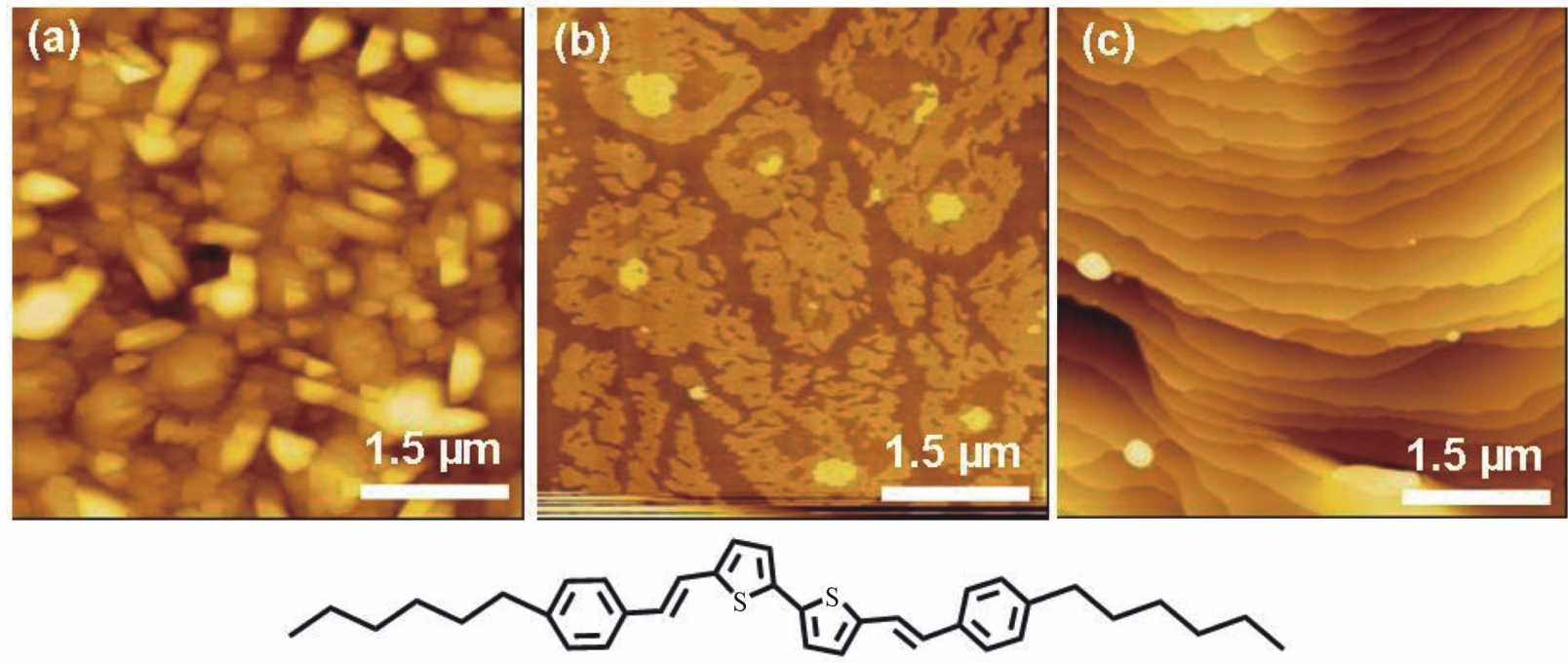

Figure 10. AFM images of DH-DS2T-based thin films deposited on $\mathrm{Si} / \mathrm{SiO}_{2}$ substrates by vapor (a), spin-coating (b) and drop-casting (c) in air at $T_{\text {sub }}=$ room temperature [21]. Molecular structure of DH-DS2T.

species, and enhanced regularity and purity, more recently it has also been the advancement of physicochemical aspects, notably materials processing, and the inducement of increased order and control of the solid state structure. Here we have shown that a fundamental understanding of the latter issues is critical to realize the full intrinsic potential that organic semiconductors possess. A complete approach including molecular structure, electrical performances and solid state morphology as well as device design is required to provide unique technologies and generate new applications.

\section{REFERENCES}

[1] G. Binnig, C. F. Quate and Ch Geber, "Atomic Force Microscope," Physical Review Letters, Vol. 56, No. 9, 1986, pp. 930-933. doi:10.1103/PhysRevLett.56.930

[2] A. Facchetti, M.-H. Yoon and T. J. Marks, "Gate Dielectrics for Organic Field-Effect Transistors: New Opportunities for Organic Electronics," Advanced Materials, Vol. 17, No. 14, 2005, pp. 1705-1725. doi:10.1002/adma.200500517

[3] T. W. Kelley, P. F. Baude, C. Gerlach, D. E. Ender, D. Muyres, M. A. Haase, D. E. Vogel and S. D. Theiss, "Recent Progress in Organic Electronics: Materials, Devices, and Processes," Chemistry of Materials, Vol. 16, No. 23, 2004, pp. 4413-4422. doi:10.1021/cm049614j

[4] S. R. Forrest, "The Path to Ubiquitous and Low-Cost Organic Electronic Appliances on Plastic," Nature, Vol. 428, No. 6986, 2004, pp. 911-918. doi:10.1038/nature 02498

[5] B. Lucas, T. Trigaud and C. Videlot-Ackermann, "Organic Transistors and Phototransistors Based on Small Molecules," Polymer International Journal (In Focus), Vol. 61, No. 3, 2012, pp. 374-389.

[6] H. Klauk, M. Halik, U. Zschieschang, G. Schmid, W.
Radlik and W. Weber, "High-Mobility Polymer Gate Dielectric Pentacene Thin Film Transistors," Journal of Applied Physics, Vol. 92, No. 9, 2002, pp. 5259-5263. doi:10.1063/1.1511826

[7] H. Yanagisawa, T. Tamaki, M. Nakamura and K. Kudo, "Structural and Electrical Characterization of Pentacene Films on $\mathrm{SiO}_{2}$ Grown by Molecular Beam Deposition," Thin Solid Films, Vol. 464-465, 2004, pp. 398-402. doi:10.1016/j.tsf.2004.06.065

[8] C. Videlot-Ackermann, J. Ackermann, H. Brisset, K. Kawamura, N. Yoshimoto, P. Raynal, A. El Kassmi and F. Fages, " $\alpha, \omega$-Distyryl-Oligothiophenes: High Mobility Semiconductors for Environmentally Stable Organic-Thin Film Transistors," Journal of the American Chemical Society, Vol. 127, No. 47, 2005, pp. 16346-16347. doi: $10.1021 / \mathrm{ja} 054358 \mathrm{c}$

[9] Y. Didane, G. H. Mehl, A. Kumagai, N. Yoshimoto, C. Videlot-Ackermann and H. Brisset, "A 'Kite' Shaped Styryl End-Capped Benzo[2,1-b:3,4-b']Dithiophene with High Electrical Performances in Organic Thin Film Transistors," Journal of the American Chemical Society, Vol. 130, No. 52, 2008, pp. 17681-17683. doi: $10.1021 / \mathrm{ja} 807504 \mathrm{k}$

[10] C. Videlot-Ackermann, J. Zhang, J. Ackermann, H. Brisset, Y. Didane, P. Raynal, A. El Kassmi and F. Fages, "PType and n-Type Quaterthiophene Based Semiconductors for Thin Film Transistors Operating in Air?" Current Applied Physics, Vol. 9, No. 1, 2009, pp. 26-33. doi:10.1016/j.cap.2007.10.087

[11] A. Facchetti, M. Mushrush, H. E. Katz and T. J. Marks, "N-Type Building Blocks for Organic Electronics: A Homologous Family of Fluorocarbon-Substituted Thiophene Oligomers with High Carrier Mobility," Advanced Materials, Vol. 15, No. 1, 2003, pp. 33-38. doi:10.1002/adma.200390003

[12] C. Videlot-Ackermann, A. K. Diallo, H. Brisset, F. Fages, F. Serein-Spirau, J.-P. Lère-Porte, A. Kumagai and N. Yoshimoto, "Growth and Morphology Properties of Bis 
(2-Phenylethynyl) End-Substituted Oligothiophenes Based Thin Films," Journal of Optoelectronics and Advanced Materials, Vol. 4, No. 10, 2010, pp. 699-704.

[13] A. K. Diallo, C. Videlot-Ackermann, P. Marsal, H. Brisset, F. Fages, A. Kumagai, N. Yoshimoto, F. Serein-Spirau and J.-P. Lère-Porte, "Acetylenic Spacers in Phenyl End-Substituted Oligothiophene Core for Highly AirStable Organic Field-Effect Transistors," Physical Chemistry Chemical Physics, Vol. 12, No. 15, 2010, pp. 38453851. doi:10.1039/b923352k

[14] L. Rapp, F. Serein-Spirau, J.-P. Lère-Porte, A. P. Alloncle, P. Delaporte, F. Fages and C. Videlot-Ackermann, "Laser Printing of Air-Stable High Performing Organic Thin Film Transistors," Organic Electronics, Vol. 13, No. 10, 2012, pp. 2035-2041. doi:10.1016/j.orgel.2012.06.020

[15] C. Videlot-Ackermann, H. Brisset, J. Zhang, J. Ackermann, S. Nénon, F. Fages, P. Marsal, T. Tanisawa and N. Yoshimoto, "Influence of Phenyl Perfluorination on Charge Transport Properties of Distyryl-Oligothiophenes in Organic Field-Effect Transistors," The Journal of Physical Chemistry C, Vol. 113, No. 4, 2009, pp. 1567-1574. doi:10.1021/jp8049262

[16] R. P. Ortiz, H. Brisset and C. Videlot-Ackermann, "Perfluoroarene Units in Distyryl-Oligothiophene Analogues: An Efficient Electron Density Confinement Preventing N-Type Transport in Organic Thin Film Transistors," Synthetic Metals, Vol. 162, No. 9-10, 2012, pp. 857-861. doi:10.1016/j.synthmet.2012.03.008
[17] M. L. Tang and Z. Bao, "Halogenated Materials as Organic Semiconductors," Chemistry of Materials, Vol. 23, No. 3, 2011, pp. 446-455. doi:10.1021/cm102182x

[18] Y. Jung, K.-J. Baeg, D.-Y. Kim, T. Someya and S. Y. Park, "A Thermally Resistant and Air-Stable N-Type Organic Semiconductor: Naphthalene Diimide of 3,5-Trifluoromethyl Aniline," Synthetic Metals, Vol. 159, No. 19-20, 2009, pp. 2117-2121. doi:10.1016/i.synthmet.2009.08.004

[19] Y. Didane, R. P. Ortiz, J. Zhang, K. Aosawa, T. Tanisawa, H. Aboubakr, F. Fages, J. Ackermann, N. Yoshimoto, H. Brisset and C. Videlot-Ackermann, "Towards N-Channel Organic Thin Film Transistors Based on a Distyryl-Bithiophene Derivative," Tetrahedron, Vol. 68, No. 24, 2012, pp. 4664-4671. doi:10.1016/j.tet.2012.04.020

[20] Y. Didane, P. Marsal, F. Fages, A. Kumagai, N. Yoshimoto, H. Brisset and C. Videlot-Ackermann, "Core-Cyanated Distyryl-Bithiophene: Synthesis and Impact on Charge Transport in Field Effect Transistors," Thin Solid Films, Vol. 519, No. 2, 2010, pp. 578-586. doi:10.1016/j.tsf.2010.07.005

[21] Y. Didane, C. Martini, M. Barret, S. Sanaur, P. Collot, J. Ackermann, F. Fages, A. Suzuki, N. Yoshimoto, H. Brisset and C. Videlot-Ackermann, "Comparison of P-Channel Transistors Based on $\alpha, \omega$-Hexyl-Distyryl-Bithiophene Prepared Using Various Film Deposition Methods," Thin Solid Films, Vol. 518, No. 18, 2010, pp. 5311-5320. doi:10.1016/j.tsf.2010.03.079 